\title{
Adolescent immaturity in attention-related brain engagement to emotional facial expressions
}

\author{
Christopher S. Monk, ${ }^{\text {a,b,* Erin B. McClure, }}{ }^{\mathrm{a}, \mathrm{b}}$ Eric E. Nelson, ${ }^{\mathrm{a}, \mathrm{b}}$ Eric Zarahn, ${ }^{\mathrm{c}}$ \\ Robert M. Bilder, ${ }^{\mathrm{d}}$ Ellen Leibenluft, ${ }^{\mathrm{b}, \mathrm{e}}$ Dennis S. Charney, ${ }^{\mathrm{b}}$ \\ Monique Ernst, ${ }^{\mathrm{a}, \mathrm{b}}$ and Daniel S. Pine ${ }^{\mathrm{a}, \mathrm{b}}$ \\ ${ }^{a}$ Section on Development and Affective Neuroscience, National Institute of Mental Health, National Institutes of Health, \\ Department of Health and Human Services, Bethesda, MD 20892, USA \\ ${ }^{\mathrm{b}}$ Mood and Anxiety Disorders Program, National Institute of Mental Health, National Institutes of Health, \\ Department of Health and Human Services, Bethesda, MD 20892, USA \\ ${ }^{\mathrm{c}}$ Department of Psychiatry, Columbia University, New York, NY 10032, USA \\ ${ }^{\mathrm{d}}$ Department of Psychiatry and Biobehavioral Sciences, University of California \\ at Los Angeles School of Medicine, Los Angeles, CA, 90095, USA \\ ${ }^{\mathrm{e}}$ Pediatric and Developmental Neuropsychiatry Branch, National Institute of Mental Health, National Institutes of Health, \\ Department of Health and Human Services, Bethesda, MD 20892, USA
}

Received 21 February 2003; revised 5 May 2003; accepted 6 June 2003

\begin{abstract}
Selective attention, particularly during the processing of emotionally evocative events, is a crucial component of adolescent development. We used functional magnetic resonance imagining (fMRI) with adolescents and adults to examine developmental differences in activation in a paradigm that involved selective attention during the viewing of emotionally engaging face stimuli. We evaluated developmental differences in neural activation for three comparisons: (1) directing attention to subjective responses to fearful facial expressions relative to directing attention to a nonemotional aspect (nose width) of fearful faces, (2) viewing fearful relative to neutral faces while attending to a nonemotional aspect of the face, and (3) viewing fearful relative to neutral faces while attention was unconstrained (passive viewing). The comparison of activation across attention tasks revealed greater activation in the orbital frontal cortex in adults than in adolescents. Conversely, when subjects attended to a nonemotional feature, fearful relative to neutral faces influenced activation in the anterior cingulate more in adolescents than in adults. When attention was unconstrained, adolescents relative to adults showed greater activation in the anterior cingulate, bilateral orbitofrontal cortex, and right amygdala in response to the fearful relative to neutral faces. These findings suggest that adults show greater modulation of activity in relevant brain structures based on attentional demands, whereas adolescents exhibit greater modulation based on emotional content.
\end{abstract}

(C) 2003 Elsevier Inc. All rights reserved.

\section{Introduction}

In a given moment in time, attention can be directed to only a small proportion of information available to the eye (Desimone, 1996). The prioritizing of stimuli for in-depth processing relies on both goal-directed (top-down) and stimulus-driven (bottom-up) attention (Corbetta and Shul-

\footnotetext{
* Corresponding author. 15K North Drive, Room 204, MSC 2670 Bethesda, MD 20892-2670. Fax: +1-301-402-2010.

E-mail address: christopher.monk@nih.gov (C.S. Monk).
}

man, 2002; Desimone and Duncan, 1995). For example, selectively attending to a spatial location requires goaldirected attention; in contrast, emotion-based stimuli, such as fearful faces, can capture attention, even if such stimuli lie outside of an attended spatial locale (Vuilleumier et al., 2001). Understanding the interactions between goal-directed and stimulus-driven processes may inform current theories of adolescent development, particularly under conditions in which emotions are aroused. Successful transition in humans from adolescence to adulthood is thought to involve maturing capacity to maintain goal-directed atten- 
tion when emotionally evocative, attention-grabbing events occur, particularly in social domains. Such maturation in the ability to maintain attention during adolescence is thought to reflect aspects of brain development (Dahl, 1996; Nelson et al., 2002; Spear, 2000). Nevertheless, the evidence to support this hypothesis remains relatively indirect. Most importantly, as of this writing, no studies have examined differences between adolescents and adults in the degree to which specific brain regions can be engaged by conditions that require maintenance of goal-directed attention during the presentation of emotional stimuli or events.

Among adults, a recent series of imaging studies suggest that the anterior cingulate cortex (ACC), orbitofrontal cortex (OFC), and amygdala are involved in attention allocation during the viewing of emotionally evocative stimuli (Adolphs, 2001; Critchley et al., 2000; Lane et al., 1997; Morris et al., 1996; Vuilleumier et al., 2001). Stimulusdriven attention influences activation within these brain structures, and fearful faces appear to be particularly effective at activating them (Morris et al., 1998; Morris et al., 1996, 1999; Whalen et al., 1998). Increased intensity of another negative expression, angry faces, is associated with enhanced ACC and OFC activity (Blair et al., 1999). Goaldirected attention also appears to modulate activity within these brain structures. For example, greater activation in the ACC emerges when adults view evocative pictures and attend to their own subjective emotional states rather than to physical features of such pictures (Lane et al., 1997). The amygdala is activated when adults perform a perceptual matching task of fearful and angry faces, but activation in the amygdala is reduced in response to another attention task in which subjects labeled the expressions of the face stimuli (Hariri et al., 2000). Further, a recent investigation documented activation of the amygdala, ACC, and OFC when attention was directed to fearful faces but not when attention was directed away from these stimuli (Pessoa et al., 2002). Taken together, available imaging data suggest that attention tasks modulate engagement of ACC, OFC, and amygdala when adults view emotionally evocative attention-grabbing stimuli.

Turning to development, few studies have examined agerelated differences in brain engagement to emotional stimuli. Available data focus on fearful faces and suggest that such stimuli engage the amygdala under some circumstances in pediatric samples (Baird et al., 1999; Killgore et al., 2001; Thomas et al., 2001). However, none of these studies manipulated attention allocation. These studies also relied on block designs and behavioral responses were not recorded during image acquisition. By varying the emotional content of the experimental condition only after multiple trials, block designs do not permit the evaluation of brain engagement with moment-to-moment changes in emotion state. In addition, when behavioral responses are not recorded during image acquisition, it is not possible to document the degree to which subjects engage in a specific psychological task. Furthermore, when comparing across groups, behavioral measures can be used in the brain-imaging analysis to examine the degree to which differences in behavioral responses mediate group differences in brain activation.

The aim of the present study was to examine developmental differences in brain engagement in a paradigm that involved performing one task in which the goal was to direct attention to internal subjective state while viewing a series of various facial expressions and performing another task in which the goal was to direct attention to an external physical feature on the same series of faces. This involves alternating between attending to and ignoring emotional aspects of these stimuli. It must be kept in mind that these directed goals are embedded in a context of overall goals for each subject that include minimizing head movement, watching the stimulus display as well as other idiosyncratic goals of each subject at a given moment.

Because the neural substrates for the processing of fearful faces is particularly well delineated in children and adults, our hypotheses focused on activation related to the response to this emotion. Nevertheless, because a high repetition of fearful faces has been shown to produce neural habituation (Fischer et al., 2003), it is advantageous to examine the neural response to fearful in the context of the presentation of other emotions. Thus, happy and angry faces were included to increase the likelihood of observing a neural response to the fearful faces. In addition, the paradigm permitted preliminary exploration of the specificity of the neural responses to the different facial expressions. We hypothesized that the neural effects of goal-directed attention would differ between adults and adolescents when they viewed fearful faces. In particular, adults are thought to better use goal-directed attention during the processing of emotionally evocative events (Csikszentmihalyi and Larson, 1984; Larson et al., 1980; Nelson et al., 2002). Therefore, we predicted that attention demands would induce greater modulation of ACC, OFC, and amygdala activation in adults relative to adolescents while viewing fearful faces. Conversely, as adolescents are hypothesized to be less likely to engage in goal-directed attention while distracting, emotionbased stimuli are presented (Csikszentmihalyi and Larson, 1984; Larson et al., 1980; Nelson et al., 2002), we predicted that fearful relative to neutral faces would induce greater activation in these structures among adolescents when subjects were instructed to attend to nonemotional facial features (nose width) or when attention was unconstrained (passive viewing). Such differences were hypothesized to reflect adolescents' relative inability to deploy goal-directed attention when confronted with potentially distracting emotional stimuli.

\section{Methods}

Subjects and task

Seventeen adults and 17 adolescents were scanned with a 3-tesla scanner. Adolescents were between the ages of 9 


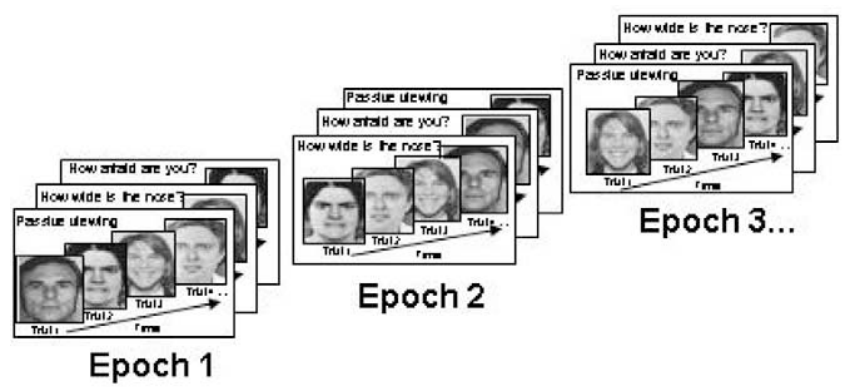

Fig. 1. Design and sample stimuli for the task. Neutral and emotional faces were presented individually. In separate blocks, subjects rated on a 1-to-5 scale either their personal subjective fear or nose width while viewing each face. There were also blocks of passive viewing. Block order was random across subjects. Neutral and emotional facial expressions varied on a random trial-by-trial basis. Fixation points were also presented to facilitate data analysis. Each block comprised 10 trials, 8 trials with faces and 2 trials with fixations. Expressions displayed by face models were also randomly varied across subjects. There were four epochs.

and 17 years $(M=13.12 ; S D=2.60)$. Such a broad age range permitted exploratory examination of developmental differences across the entire adolescent period. Adults' ages ranged from 25 to 36 years $(M=30.76 ; S D=3.07)$. There were 8 females and 9 males in each age group. All participants had age-adjusted IQs greater than 70 (adolescent $M$ $=117.41 S D=12.11$; adult $M=113.24, S D=10.38)$ and were healthy as determined by a physical examination and a standardized structured psychiatric interview (Kaufman et al., 1997; Spitzer, 1992). Participants were acclimated to the MRI environment with an MRI simulator. During simulator training, subjects were familiarized to the task with a practice set of faces and were extensively trained to achieve adequate levels of task performance. The face stimuli from the practice set were different individuals than the ones who were displayed in the actual MRI and they were all presented with neutral facial expressions.

The functional MRI (fMRI) task comprised 160 trials presented in 4 epochs and there were 4 blocks within each epoch (Fig. 1). Throughout the task, participants viewed neutral and emotional faces (angry, fearful, and happy), whereas attending either to their subjective emotional reactions or to a physical feature of the face. In one block, participants rated how afraid they felt while viewing each face (subjective emotional reactions). In a second block, participants rated the nose width on each face (physical feature). In a third block, subjects passively viewed faces; this condition allows examination of brain activation under conditions in which attention is minimally constrained. In the fourth block, subjects rated hostility of the face (not discussed in this article). In subsequent epochs, subjects viewed the same faces, but made other ratings. Blocks were randomly ordered across subjects. For each block, instructions were presented for $3 \mathrm{~s}$ and each face was displayed for $4 \mathrm{~s}$. We chose this relatively long presentation duration for the faces, because we wanted to ensure that subjects made their rating "online" while viewing the face, so that the rating would not involve their memory of the stimulus or the subjective experience. Two trials with fixation points were displayed randomly in each block for $4 \mathrm{~s}$ to facilitate data analysis. Following each face and fixation trial, there was an interstimulus interval that randomly varied between 750 and $1250 \mathrm{~ms}$. Gray-scale face stimuli were derived from Ekman and Friesen (1976), Gur (www.uphs.upenn.edu/bbl/pubs/ downloads/nptasks.shtml), and Tottenham and Nelson (www.macbrain.org/faces/index.htm). For a given subject, 32 actors presented four facial expressions (happy, angry, fearful, and neutral) with 8 different actors presenting each expression. These 32 actors were randomly selected from a larger pool of 56 actors for each subject. Similarly, the selection of facial expression for a given actor also randomly varied across subjects such that different actors displayed different emotions to different participants. Stimuli were displayed on the Avotec Silent Vision Glasses (Stuart, FL) and the five-key button box was developed by MRI Devices (Waukesha, WI).

\section{Imaging and data analysis}

Imaging was conducted on a General Electric Signa 3-tesla scanner. A sagittal localizer scan was acquired to orient subsequent scans. Functional imaging scans involved a series of 23 contiguous 5-mm axial slices covering the entire brain, parallel to the AC-PC. These scans used an echo-planar single shot gradient echo $\mathrm{T} 2 *$ weighting $(\mathrm{TR}=$ $2000 \mathrm{~ms} ; \mathrm{TE}=40 \mathrm{~ms} ; \mathrm{FOV}=240 \mathrm{~mm} ; 64 \times 64$ matrix , $3.75 \mathrm{~mm}$ voxels). High-resolution T1-weighted volumetric scans used a magnetization prepared gradient echo sequence (MP-RAGE) [180 $1.0 \mathrm{~mm}$ sagittal slices; FOV $=256 \mathrm{~mm}$, $\mathrm{NEX}=1, \mathrm{TR}=11.4 \mathrm{~ms}$, TE $=4.4 \mathrm{~ms}$; matrix $=256 \times$ 256; $\mathrm{TI}=300 \mathrm{~ms}$, bandwidth $130 \mathrm{~Hz} /$ pixel $=33 \mathrm{kHz}$ for 256 pixels in-plane resolution $=1 \mathrm{~mm}^{3}$ ].

Functional imaging data were analyzed using SPM99 with an event-related model. Definitions of ACC, OFC, and amygdala follow established procedures (Drevets and Raichle, 1998; Szeszko et al., 1999; Vogt, 1993). In particular, as described in Szeszko et al. (1999), the boundaries of the amygdala comprised the slice at the same plane as the mammillary bodies to the anterior limit of the amygdala. The OFC boundaries were defined as the last slice with the anterior horizontal ramus, the last slice containing the olfactory sulcus, anterior horizontal ramus, and the olfactory sulcus. The ACC boundaries were the tip of the cingulate sulcus, connection of the superior and precentral sulci, callosal sulcus, and cingulate sulcus. Functional data were corrected for slice timing, motion corrected, coregistered to the anatomical data, aligned to the first volume for each subject, spatially normalized to a Montreal Neurological Institute (MNI) T1-weighted template image supplied with SPM99. Recently, transformations of brain images to a common stereotactic space were found to be consistent between adults and juveniles as young as 7 years (Burgund et al., 2002). The images were then smoothed with an 8-mm 
Table 1

Mean (SD) fear rating responses

\begin{tabular}{lllll}
\hline & Happy & Neutral & Fear & Angry \\
\hline Adolescents & $1.03(0.09)$ & $1.47(0.61)$ & $1.76(0.67)$ & $2.17(0.84)$ \\
Adults & $1.03(0.12)$ & $1.71(0.66)$ & $2.59(0.89)$ & $2.98(1.11)$
\end{tabular}

Note. The range of the scale was $1-5$, where 1 represented no fear and 5 indicated extreme fear.

FWHM Gaussian kernel. Within-subject time series modeling accounted for the following conditions: face type (neutral, happy, fearful, and angry) and task (fear rating, nose width rating, passive viewing, and hostile rating). The fMRI response to each event type in each instruction condition was modeled as a rectangular pulse (of $4 \mathrm{~s}$ duration) convolved with the hemodynamic response function specified in SPM99 (default parameters). Instruction periods were similarly modeled. From each participant, contrast images were generated for the pairwise comparison of the mean BOLD signal for each condition.

To examine between-group effects, the contrast images from each subject were fit to a second-level random effects analysis of age (adolescents vs adults). Brains were intensity normalized and smoothed at 11.4 full-width half-maximum. For the analysis of the ACC and OFC, we applied a correction for multiple comparisons across the small volume (Worsley et al., 1996). We report activations that survive $P$ $<0.05$ corrected per region. However, because the small volume correction approach relies on smoothed data, small structures such as the amygdala might suffer from appreciable partial volume effects. Therefore, for the analysis of the amygdala activation, the mean contrast values were calculated for the entire left and right amygdalae separately from the unsmoothed data. Contrast values from each amygdala were computed for each subject for a given comparison. The amygdala ROI was drawn from the MNI template and individual subject brains were normalized to the MNI brain.

Behavioral results for fear and nose ratings as well as reaction times were submitted to a repeated-measures ANOVA. Button press responses that were less than 2.5 SD from the mean reaction time were excluded. Responses that occurred after $4 \mathrm{~s}$ (i.e., at the end of the trial) were not recorded. A Greenhouse-Geisser correction for sphericity was used when necessary.

In a post hoc analysis, we examined the degree to which age differences in behavioral measures accounted for differences in the fMRI signal. To accomplish this, we used a regression model to define slopes for the adults and adolescents based on the behavioral ratings. The behavioral ratings were treated as covariates and we assessed age differences at comparable points on the two slopes. The rating of 2 for subjective fear level acted as the comparable point for the two age groups. The 2 rating was chosen because it was the most common response for subjects in which any increased level of fear is provided.
Further, the broad age range of the adolescent group permitted exploratory analyses to be performed on the relationship between voxels that yielded activation in the primary analyses of interest and pediatric age in years. (As with other analyses with the amygdala, the entire ROI rather than the voxels was used.) Spearman's correlations were employed to examine the relationship of peak voxels for each subject within contrasts in which a developmental effect was documented and age in years.

\section{Results}

\section{Behavioral results}

The mean reported fear levels for adolescents and adults are presented in Table 1. The same ordering of induced fear was seen across age groups, from happy to angry facial displays. A repeated-measures ANOVA with a Greenhouse-Geisser correction (this correction was used for all subsequent ANOVAs) indicated that there was a significant difference in behavioral subjective fear ratings among facial expression $[F(3,96)=55.45, P<.001]$, collapsed across age group. These results confirm that subjects' emotional responses to the faces were as predicted, with fear levels increasing as facial expressions transitioned from positive to negative. In addition, there was a significant interaction of facial expression and age group $[F(3,96)=5.28, P<.01]$. Thus, whereas both age groups exhibited appropriate changes in fear levels, adults endorsed greater fear to the faces.

The pattern for the nose width ratings was markedly different from the fear ratings. Moreover, the pattern was comparable in adolescents and adults, suggesting that both adolescents and adults correctly followed differential instructions across the two rating conditions. The mean nose width ratings for adolescents and adults are presented in Table 2. There was a significant effect of facial expression on nose width rating $[F(3,96)=14.88, P<.001]$ and no age group interaction $[F(3,96)=0.73, P=.535]$. Variation in nose width rating due to facial expression was anticipated, because these expressions often involve broadening of the nostrils. The finding that there were no interactions of age group on the nose width ratings suggests that adults and adolescents performed the tasks comparably.

Reactions times for adolescents to fear rating in milli-

Table 2

Mean (SD) nose width ratings responses

\begin{tabular}{lllll}
\hline & Happy & Neutral & Fear & Angry \\
\hline Adolescents & $2.52(0.37)$ & $2.11(0.49)$ & $2.16(0.49)$ & $2.46(0.45)$ \\
Adults & $2.46(0.51)$ & $2.13(0.61)$ & $2.25(0.59)$ & $2.61(0.49)$
\end{tabular}

Note. The range of the scale was $1-5$, where 1 indicated that the nose was not wide and 5 was used to report that the nose was extremely wide. 
Table 3

Regions of activation across development

\begin{tabular}{|c|c|c|c|c|c|c|}
\hline & $x$ & $y$ & $z$ & $t_{30}$ score & Cluster size & Region \\
\hline \multicolumn{7}{|c|}{ Adults minus adolescents contrasts* } \\
\hline $\begin{array}{l}\text { Subjective fear to fearful faces } \\
\text { minus nose width to fearful } \\
\text { faces }\end{array}$ & 40 & 22 & -18 & 3.22 & 530 & Right OFC \\
\hline \multicolumn{7}{|c|}{ Adolescents minus adult contrasts** } \\
\hline $\begin{array}{l}\text { Nose width to fearful faces } \\
\text { minus nose width to neutral } \\
\text { faces }\end{array}$ & 16 & 46 & 16 & 4.18 & 1189 & $\mathrm{ACC}$ \\
\hline Passive viewing to fearful faces & -2 & 30 & 30 & 3.92 & 1631 & $\mathrm{ACC}$ \\
\hline minus passive viewing to & -4 & 28 & 40 & 3.55 & & $\mathrm{ACC}$ \\
\hline neutral faces & -2 & 14 & 26 & 3.51 & & $\mathrm{ACC}$ \\
\hline & 4 & 14 & 24 & 3.47 & & $\mathrm{ACC}$ \\
\hline & 4 & 10 & 26 & 3.42 & & $\mathrm{ACC}$ \\
\hline & -2 & 10 & 28 & 3.41 & & $\mathrm{ACC}$ \\
\hline & -4 & 10 & 42 & 3.37 & & $\mathrm{ACC}$ \\
\hline & -30 & 26 & -2 & 3.70 & 1077 & Left OFC \\
\hline & -24 & 22 & -18 & 3.49 & & Left OFC \\
\hline & 22 & 22 & -10 & 3.39 & 274 & Right OFC \\
\hline & 22 & 18 & -14 & 3.08 & & $\begin{array}{l}\text { Right OFC } \\
\text { Right Amyg }\end{array}$ \\
\hline
\end{tabular}

Note. For both Tables 1 and 2, all voxelwise $t$ values are significant at $P<0.05$ corrected for multiple comparisons within each region and coordinates are reported in MNI space (Collins et al., 1998).

* For the contrasts with developmental effects, adults alone also showed activation differences across task conditions in the same regions. In addition, adolescents alone showed no activation differences across task conditions within the ROIs.

** In addition to these developmental effects displayed in these contrasts, adolescents alone revealed activation differences across task conditions in the same areas and adults alone did not reveal differential activation across task conditions in the ROIs.

seconds were 1367.29 (332.06) for happy, 1729.40 (394.16) for neutral, 1782.47 (395.11) for fear, and 1905.01 (355.91) for angry. Among adults, the reaction times for fear ratings were 1280.37 (209.75) for happy, 1658.61 (467.24) for neutral, 1963.85 (374.35) for fear, and 2021.98 (374.55) for angry. A repeated-measures ANOVA demonstrated that facial expression influenced reaction time $[F(3,96)=45.08$, $P<.001]$. The interaction for age and facial expression did not reach significance $[F(3,96)=2.54, P=.069]$. Although the interaction was not significant, this trend for an age by facial expression interaction suggests that changes of emotional content within the faces differentially influences ongoing psychological processes in adolescents and adults. Reaction times for nose width rating for adolescents were as follows: 1961.72 (419.66) for happy, 1776.22 (299.14) for neutral, 1894.69 (318.19) for fear, 2007.18 (345.99) for angry. For adults, the reaction times were as follows: 1889.22 (324.78) to happy, 1803.32 (276.60) to neutral, 1878.65 (346.03) to fear, and 1958.64 (350.35) to angry. A repeated-measures ANOVA showed that facial expression affected reaction time response $[F(3,96)=5.25$, $P<.005]$. There were no interactions with age $[F(3,96)=$ $0.37, P=.78]$. This suggests that emotional expressions were distracting, in that reaction times were faster when viewing neutral faces than when viewing emotional faces. Moreover, the emergence of similar data in adults and adolescents suggests the two groups were performing the task similarly.

\section{Imaging results}

Consistent with our hypothesis, attention demands (attending to subjective fear relative to attending to an external physical feature) induced greater modulation of activation in response to fearful faces in the right OFC in adults relative to adolescents (see Table 3; Fig. 2A ). The nature of this between-group difference was explored further by examining activation profiles in each age group separately. In this analysis, adults showed significant brain activation differences between the two attention conditions in response to the fearful faces in the same location of the right OFC. Adolescents, in contrast, showed no differential response for any ROI in these comparisons across attention tasks.

We also confirmed the hypothesis that emotional content would induce greater activation in the ACC, OFC, and amygdala among adolescents when subjects were instructed to attend to nonemotional facial features (nose width) or when attention was unconstrained (passive viewing). Specifically, when focusing attention on nose width, we found that adolescents, relative to adults, exhibited greater activation in the ACC in response to fearful faces (Table 3; Fig. 2B). Again, the nature of these between-group differences 
A

2

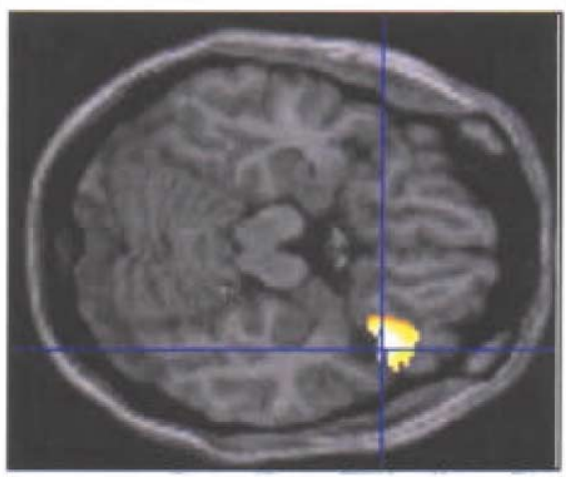

B

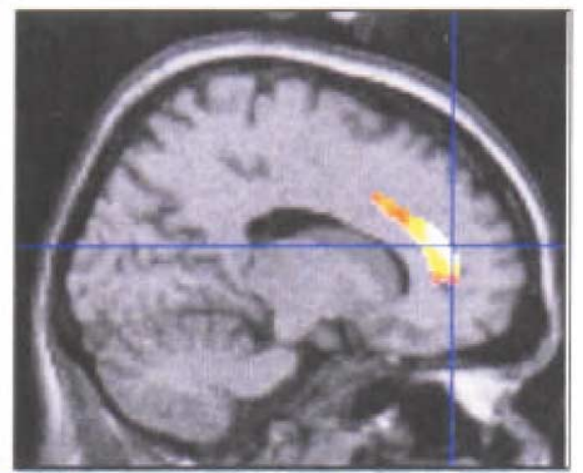

A

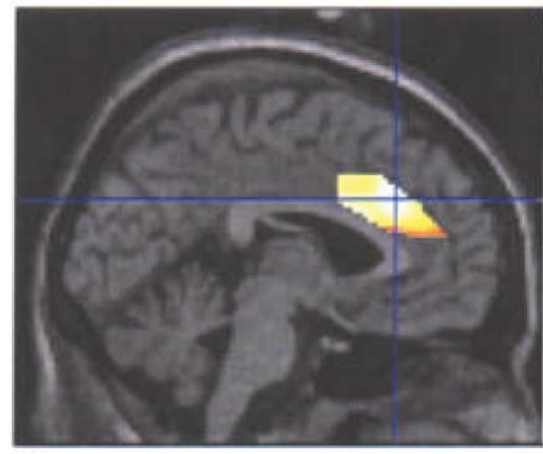

C

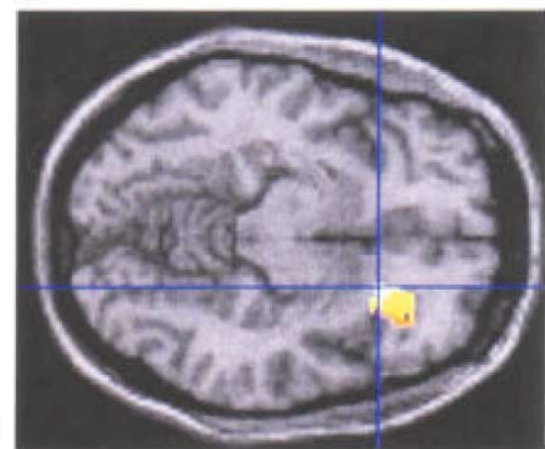

B

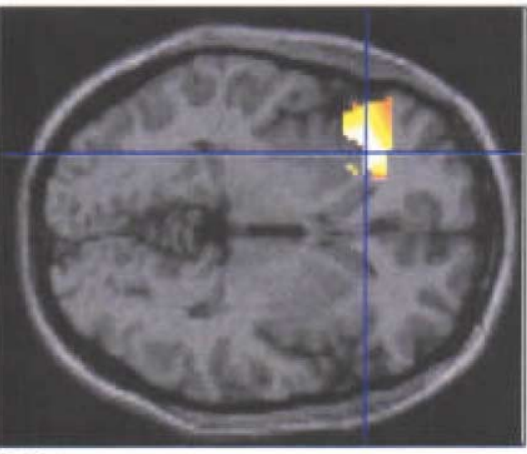

D

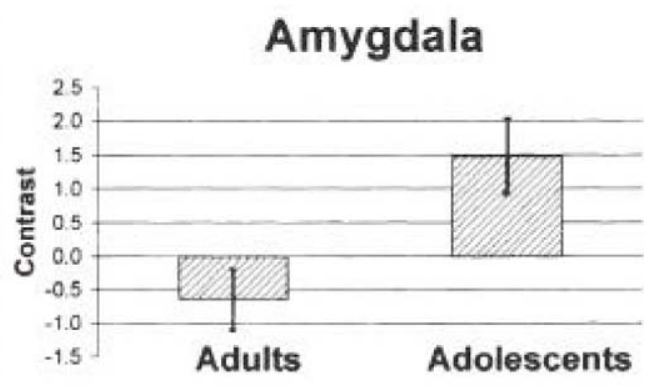

Fig. 2. Developmental changes in brain activation. When attending to subjective fear responses compared to attending to a physical feature (nose width), adults displayed greater activation relative to adolescents in the right $\mathrm{OFC}$ while viewing fearful faces (A). When attending to a nonemotional physical feature, adolescents showed greater ACC activation than adults to fearful relative to neutral face (B). Crosshairs indicate cluster maxima. Analyses were restricted to the ROIs as described in the text. In figures, we emphasize activations to fearful face stimuli since most of the previous neuroimaging work on face processing in juveniles and adults examined fear (Breiter et al., 1996; Morris et al., 1996; Pessoa et al., 2002; Thomas et al., 2001; Whalen et al., 1998). Fig. 3. Activation patterns in adolescents relative to adults when passively viewing fearful minus neutral faces. (A-D) Activation in the ACC, left OFC, right OFC, and amygdala, respectively. Crosshairs indicate cluster maxima. Analyses were restricted to the ROIs described as in the text.

was explored by examining activation profiles in each age group separately. Adolescents alone displayed activation differences in the same location of the ACC. Adults alone showed no activation in these contrasts. Moreover, during passive viewing, adolescents compared to adults showed a greater response of the ACC, OFC, and the right amygdala to fearful vs neutral faces (Table 3; Fig. 3A-D ). Adolescents alone showed significant activation in each of these locations in this contrast. Adults did not respond differen- tially to fearful vs neutral faces in the passive viewing condition. Therefore, when instructed to attend to a nonemotional feature of the face, or when attention was unconstrained (passive viewing), the fearful expression influenced neural activity more strongly in adolescents than in adults.

The main unanticipated fMRI result was the lack of amygdala activation to the passive viewing of fearful faces among adults (Fig. 3). This finding is described further in the Discussion. 
Table 4

Regions of activation across development for the post hoc analyses involving the angry, happy, and neutral face stimuli

\begin{tabular}{|c|c|c|c|c|c|c|}
\hline & $x$ & $y$ & $z$ & $\mathrm{t}_{30}$ score & Cluster size & Region \\
\hline \multicolumn{7}{|c|}{ Adults minus adolescents contrasts* } \\
\hline Subjective fear to angry faces & 18 & 38 & 4 & 3.39 & 359 & $\mathrm{ACC}$ \\
\hline minus nose width to angry & 14 & 36 & 4 & 3.30 & & $\mathrm{ACC}$ \\
\hline faces & -22 & 34 & 6 & 2.94 & 77 & Left OFC \\
\hline $\begin{array}{l}\text { Subjective fear to happy faces } \\
\text { minus nose width to happy } \\
\text { faces }\end{array}$ & -24 & 28 & -4 & 3.13 & 569 & Left OFC \\
\hline $\begin{array}{l}\text { Subjective fear to neutral faces } \\
\text { minus nose width to neutral } \\
\text { faces }\end{array}$ & & & & ns & & \\
\hline \multicolumn{7}{|c|}{ Adolescents minus adult contrasts** } \\
\hline $\begin{array}{l}\text { Nose width to angry faces } \\
\text { minus nose width to neutral }\end{array}$ & & & & ns & & \\
\hline $\begin{array}{l}\text { Nose width to happy faces } \\
\text { minus nose width to neutral }\end{array}$ & -28 & 30 & -18 & 3.04 & 764 & Left OFC \\
\hline $\begin{array}{l}\text { Passive viewing to angry faces } \\
\text { minus passive viewing to } \\
\text { neutral faces }\end{array}$ & & & & ns & & \\
\hline $\begin{array}{l}\text { Passive viewing to happy faces } \\
\text { minus passive viewing to } \\
\text { neutral faces }\end{array}$ & & & & ns & & \\
\hline
\end{tabular}

* For the contrasts with developmental effects, adults alone also showed activation differences across task conditions in the same regions. In addition, adolescents alone showed no activation differences across task conditions within the ROIs.

** In addition to these developmental effects displayed in these contrasts, adolescents alone revealed activation differences across task conditions in the same areas and adults alone did not reveal differential activation across task conditions in the ROIs.

\section{Post hoc analyses}

Exploratory analyses were performed on the neural response to angry and happy facial expressions. For the attention tasks of subjective fearful relative to external physical feature, angry faces were associated with increased ACC and left OFC for the adults compared to the adolescents (Table 4). In the same comparison of attention tasks, happy faces related to increased left OFC in adults relative to adolescents (Table 4). Finally, these effects were apparent only with emotional and not neutral facial expressions. No between-group differences emerged in activation across attention states during the viewing of neutral faces (Table 4). Hence, developmental difference in attentional processing emerges only during presentation of emotional stimuli: when viewing emotional faces, attentional task demands have a greater influence on adult than on adolescent neural activity.

When attention was directed to the nose, angry faces relative to neutral faces was not associated with differential activation in the adults or adolescents, but happy faces relative to neutral faces led to increased activation in the left OFC in the adolescents compared to adults. In the passive viewing condition, angry relative to neutral faces and happy relative to neutral faces did not yield developmental differences.

Because there were behavioral differences in the subjec- tive fear ratings between the adults and adolescents, it is possible that developmental differences in the fMRI response could be accounted for by these behavioral differences. To evaluate this possibility, we implemented a covariate analyses. Using the behavioral covariate of the rating of 2, the contrast of subjective fear rating to fearful faces minus nose width rating to fearful faces was examined and it was found that there was still activation across development, $t=2.82, P<.05$ (corrected for a $3-\mathrm{mm}$ sphere) at the same MNI coordinate $(x y z)$ of $4022-18$.

Further exploratory analyses were carried out relating peak voxel activation from the primary analyses to pediatric age in years (see Table 3 for a list of the coordinates for the peak voxels). Spearman's correlation revealed no significant relationships of age in years with the contrast of subjective fear rating to fearful faces relative to nose width rating to fearful faces, $-.032, P=.902$. Similarly, the correlation for the peak voxel and age for the contrast of nose width rating to fearful faces relative to the nose width rating to neutral faces was not significant, .116, $P=.658$. For the contrast of passive viewing of fearful faces relative to passive viewing of neutral faces, the right amygdala, the ACC, and the left as well as the right OFC showed activation in the adolescents relative to the adults and, therefore, peak voxels from each ROI were examined. Among the adolescents, there were no effects of age in the right amygdala ROI, coefficient $=-.342, P=.179$, and no relation- 
ship was found in the ACC, $-.338, p=.185$. However, a significant correlation was found in the left OFC, $-.529, P$ $<.05$, and the right OFC, $-.502, P<.05$. Finally, there was no evidence that gender modulated any of the results reported here.

\section{Discussion}

This study examined differences in brain engagement of adolescents and adults during selective attention to emotional and nonemotional features of facial expressions. Two main novel findings emerged from this study. First, goaldirected attention influenced activation in the OFC more strongly in adults than in adolescents during the viewing of fearful faces. Second, the emotional content of face stimuli appeared to drive activation more strongly in the ACC, OFC, and amygdala in adolescents than in adults. These differences emerge only under conditions when attention was not directed to processing emotion (i.e., in nose width rating and passive viewing). These findings suggest that adults modulate activity in relevant brain structures to a greater degree based on attention demands, whereas adolescents modulate activity to a greater degree based on the nature of emotion in the stimulus. Maturation between adolescence and adulthood may involve increased ability to engage relevant brain regions for goal-directed attention when emotionally evocative, attention-grabbing events occur.

Our findings in adults are consistent with the recent work of Pessoa et al. (2002). As in the current study, Pessoa et al. used a hybrid design in which facial expressions varied across trials and attention tasks varied across blocks. Pessoa et al. demonstrated that the attention task influences activation in adults in structures responsive to emotional stimuli and attention, in particular the OFC and the amygdala. Although we found no amygdala activation in adults when they attended to emotion states, these attention task demands did modulate activation of the adult OFC. Differences in activation of the amygdala in adults between the present study and the work of Pessoa et al. may be due to methodological factors. For example, whereas Pessoa et al. presented the faces for $200 \mathrm{~ms}$, the presentation duration of the present study was $4 \mathrm{~s}$, introducing the possibility that the amygdala habituated to the face stimuli in the current study. The relatively long presentation rate was chosen because we wished to ensure that subjects made the behavioral rating while viewing the face and, therefore, participants would not need to rely on memory. In addition, Pessoa et al. documented amygdala activation using a gender identification task and we did not use such a task in the current study.

Among children and adolescents, virtually no research examines the neural correlates of selective attention in the context of emotion. Up to this point, no fMRI study has explicitly examined selective attention to emotional and nonemotional features of stimuli. However, three studies selectively imaged the amygdala in children and adolescents in a task that involved the presentation of emotional facial expressions (Baird et al., 1999; Killgore et al., 2001; Thomas et al., 2001). These studies used block designs to document activation of the amygdala to fearful faces compared to fixation, though, interestingly, activation in contrasts of fearful vs neutral faces did not consistently emerge. Thus, although these studies document amygdala engagement to emotion-based stimuli that are likely to engage attention (Bradley et al., 1997; Hanson and Hanson, 1988; Ohman et al., 2001), the investigations did not explicitly evaluate the role of attention in the task. The present study builds on this previous work. Like prior studies, amygdala activation did emerge to fearful faces viewed passively among adolescents. Moreover, results in the current study raise the possibility that this response is modulated by attentional demands. Finally, and perhaps most importantly, in direct comparisons between age groups, the current study provides initial evidence that modulation of amygdala, as well as ACC and OFC, by attention demands differs between adults and adolescents

One limitation of this study is that eye gaze was not monitored. Developmental differences in eye gaze may influence differences in brain activation. An important direction for future research will be to monitor eye gaze of adolescents and adults as they view emotional faces both in and out of the MRI scanner. Such work would indicate whether developmental differences in brain activation might be mediated by variations in the locations or durations of visual fixation on the faces. A second limitation is that although there were significant differences in reported fear across facial expressions, the fear levels were relatively modest. This paradigm did not induce a highly emotionally arousing experience. Instead, this is an effective paradigm for influencing modest changes in emotion and examining the neuroanatomical correlates of those changes. The present study is also limited by a lack of a measure to verify changes in emotion. Although there is no gold standard for objectively evaluating changes in emotion state, the inclusion of a measure that attempts to index these changes, such as skin conductance, would potentially provide validation for the subjective reports.

Adolescence is associated with increased prevalence of psychopathology involving perturbation in emotion (Angold et al., 1999; Pine et al., 1998). Thus, an important direction will be to extend this work to adolescent populations at high risk for such forms of psychopathology. Findings from this work may help uncover how changes in neurophysiological function at adolescence relate to both healthy development and risk for psychopathology. Furthermore, the present study was not designed to examine the effects of puberty independent of age. Because puberty and age are so highly correlated, novel designs are needed to generate insights on specificity of associations with puberty as opposed to age (Angold et al., 1999). For example, in order to evaluate puberty, future work may select adoles- 
cents of the same age who are discordant for pubertal status (Nelson et al., 2002). Using such an approach with the present paradigm may help to reveal the role of puberty on the control of attention allocation during the viewing of emotional stimuli.

\section{Acknowledgments}

We thank K. Towbin and A. Zametkin for medical oversight and H. Iwamoto, A. Hoberman, A. Merikangas, L.A. Montgomery, and S. Munson for technical support.

\section{References}

Adolphs, R., 2001. The neurobiology of social cognition. Curr. Opin. Neurobiol. 11, 231-239.

Angold, A., Costello, E.J., Erkanli, A., Worthman, C.M., 1999. Pubertal changes in hormone levels and depression in girls. Psychol. Med. 29, 1043-1053.

Baird, A.A., Gruber, S.A., Fein, D.A., Mass, L.C., Steingard, R.J., Renshaw, P.F., Cohen, B.M., Yurgelun-Todd, D.A., 1999. Functional magnetic resonance imaging of facial affect recognition in children and adolescents. J. Am. Acad. Child Adolesc. Psychiat. 38, 195-199.

Blair, R.J.R., Morris, J.S., Frith, C.D., Perrett, D.I., Dolan, R.J., 1999 Dissociable neural responses to facial expressions of sadness and anger. Brain 122, 883-893.

Bradley, B.P., Mogg, K., Lee, S.C., 1997. Attentional biases for negative information in induced and naturally occurring dysphoria. Behav. Res. Ther. 35, 911-927.

Breiter, H.C., Etcoff, N.L., Whalen, P.J., Kennedy, W.A., Rauch, S.L., Buckner, R.L., Strauss, M.M., Hyman, S.E., Rosen, B.R., 1996. Response and habituation of the human amygdala during visual processing of facial expression. Neuron 17, 875-87.

Burgund, E.D., Kang, H.C., Kelly, J.E., Buckner, R.L., Snyder, A.Z., Petersen, S.E., Schlaggar, B.L., 2002. The feasibility of a common stereotactic space for children and adults in fMRI studies of development. Neuroimage 17, 184-200.

Collins, D.L., Zijdenbos, A.P., Kollokian, V., Sled, J.G., Kabani, N.J., Holmes, C.J., Evans, A.C., 1998. Design and construction of a realistic digital brain phantom. IEEE Trans. Med. Imag. 17, 463-468.

Corbetta, M., Shulman, G.L., 2002. Control of goal-directed and stimulusdriven attention in the brain. Nat. Rev. Neurosci. 3, 201-215.

Critchley, H.D., Elliott, R., Mathias, C.J., Dolan, R.J., 2000. Neural activity relating to generation and representation of galvanic skin conductance responses: a functional magnetic resonance imaging study. J. Neurosci. 20, 3033-3340,

Csikszentmihalyi, M., Larson, R., 1984. Being Adolescent: Conflict and Growth in the Teenage Years. Basic Books, New York.

Dahl, R.E., 1996. The regulation of sleep and arousal: development and psychopathology. Dev. Psychopathol. 8, 3-27.

Desimone, R., 1996. Neural mechanisms for visual memory and their role in attention. Proc. Natl. Acad. Sci. USA 93, 13494-13499.

Desimone, R., Duncan, J., 1995. Neural mechanisms of selective visual attention. Annu. Rev. Neurosci. 18, 193-222.

Drevets, W.C., Raichle, M.E., 1998. Reciprocal suppression of regional cerebral blood flow during emotional versus higher cognitive processes: implications for interactions between emotion and cognition. Cogn. Emot. 12, 353-385.

Ekman, P., Friesen, W.V., 1976. Pictures of Facial Affect. Consulting Psychologists Press, Palo Alto, CA.

Fischer, H., Wright, C.I., Whalen, P.J., McInerney, S.C., Shin, L.M., Rauch, S.L., 2003. Brain habituation during repeated exposure to fear- ful and neutral faces: a functional MRI study. Brain Res. Bull. 59, 387-392.

Hanson, C., Hanson, R., 1988. Finding the face in the crowd: an anger superiority effect. J. Pers. Soc. Psychol. 54, 917-924.

Hariri, A.R., Bookheimer, S.Y., Mazziotta, J.C., 2000. Modulating emotional responses: effects of a neocortical network on the limbic system. NeuroReport 11, 43-48

Kaufman, J., Birmaher, B., Brent, D., Rao, U., Flynn, C., Moreci, P., Williamson, D., Ryan, N., 1997. Schedule for affective disorders and schizophrenia for school-age children-present lifetime version $(\mathrm{K}$ SADS-PL): initial reliability and validity data. J. Am. Acad. Child Adolesc. Psychiat. 36, 980-988.

Killgore, W.D., Oki, M., Yurgelun-Todd, D.A., 2001. Sex-specific developmental changes in amygdala responses to affective faces. NeuroReport $12,427-433$.

Lane, R.D., Fink, G.R., Chau, P.M., Dolan, R.J., 1997. Neural activation during selective attention to subjective emotional responses. NeuroReport 8, 3969-3972.

Larson, R., Csikszentmihalyi, M., Graef, R., 1980. Mood variability and the psychosocial adjustment of adolescents. J. Youth Adolesc. 9, 469-490.

Morris, J.S., Friston, K.J., Buchel, C., Frith, C.D., Young, A.W., Calder, A.J., Dolan, R.J., 1998. A neuromodulatory role for the human amygdala in processing emotional facial expressions. Brain 121, 47-57.

Morris, J.S., Frith, C.D., Perrett, D.I., Rowland, D., Young, A.W., Calder, A.J., Dolan, R.J., 1996. A differential neural response in the human amygdala to fearful and happy facial expressions. Nature 383, 812-815.

Morris, J.S., Ohman, A., Dolan, R.J., 1999. A subcortical pathway to the right amygdala mediating "unseen" fear. Proc. Natl. Acad. Sci. USA $96,1680-1685$

Nelson, C.A., Bloom, F.E., Cameron, J.L., Amaral, D., Dahl, R.E., Pine, D.S., 2002. An integrative, multidisciplinary approach to the study of brain-behavior relations in the context of typical and atypical development. Dev. Psychopathol. 14, 499-520.

Ohman, A., Lundqvist, F., Esteves, F., 2001. The face in the crowd revisited: a threat advantage with schematic stimuli. J. Pers. Soc. Psychol. 80, 381-396.

Pessoa, L., McKenna, M., Gutierrez, E., Ungerleider, L.G., 2002. Neural processing of emotional faces requires attention. Proc. Natl. Acad. Sci. USA 99, 11458-11463.

Pine, D.S., Cohen, P., Gurley, D., Brook, J., Ma, Y., 1998. The risk for early-adulthood anxiety and depressive disorders in adolescents with anxiety and depressive disorders. Arch. Gen. Psychiat. 55, 56-64.

Spear, L.P., 2000. The adolescent brain and age-related behavioral manifestations. Neurosci. Biobehav. Rev. 24, 417-463.

Spitzer, R.L., Williams, J.B., Gibbon, M., First, M.B., 1992. The Structured Clinical Interview for DSM-III-R (SCID). I: history, rationale, and description. Arch. Gen. Psychiat. 49, 624-629.

Szeszko, P.R., Robinson, D., Alvir, J.M., Bilder, R.M., Lencz, T., Ashtari, M., $\mathrm{Wu}, \mathrm{H}$., Bogerts, B., 1999. Orbital frontal and amygdala volume reductions in obsessive-compulsive disorder. Arch. Gen. Psychiat. 56, 913-919.

Thomas, K.M., Drevets, W.C., Whalen, P.J., Eccard, C.H., Dahl, R.E., Ryan, N.D., Casey, B.J., 2001. Amygdala response to facial expressions in children and adults. Biol. Psychiat. 49, 309-316.

Vogt, B.A., 1993. Structural organization of cingulate cortex: Areas, neurons, and somatodendritic transmitter receptors, in: Vogt, B.A., Gabriel, M. (Eds.), Neurobiology of Cingulate Cortex and Limbic Thalamus: A Comprehensive Handbook. Birkhauser, Boston.

Vuilleumier, P., Armony, J.L., Driver, J., Dolan, R.J., 2001. Effects of attention and emotion on face processing in the human brain: an event-related fMRI study. Neuron 30, 829-841.

Whalen, P.J., Rauch, S.L., Etcoff, N.L., McInerney, S.C., Lee, M.B., Jenike, M.A., 1998. Masked presentations of emotional facial expressions modulate amygdala activity without explicit knowledge. J. Neurosci. $18,411-418$

Worsley, K.J., Marrett, S., Neelin, P., Vandal, A.C., Friston, K.J., Evans, A.C., 1996. A unified statistical approach for determining significant signals in images of cerebral activation. Hum. Brain Mapp. 4, 58-73. 